\title{
FOLIATIONS AND GROUPS OF DIFFEOMORPHISMS
}

\author{
BY WILLIAM THURSTON
}

Communicated by William Browder, July 7, 1973

John Mather has described a close relation between framed codimension-one Haefliger structures (these form a class of singular foliations), and the group of compactly supported diffeomorphisms of $\boldsymbol{R}^{1}$, with discrete topology [11], [12], [14]. In this announcement I will describe generalizations of his ideas to higher codimension Haefliger structures and groups of diffeomorphisms of arbitrary manifolds. See Haefliger [7] for a development of Haefliger structures and their classifying spaces.

I would like to thank Boyd Anderson, André Haefliger and John Mather for long discussions and proddings about this material, and many others for helpful conversations and relevant information.

Let Diff ${ }^{r}\left(M^{p}\right)$ denote the group of $C^{r}$ diffeomorphisms of $M^{p}$, a closed manifold. Let $\operatorname{Diff}_{0}^{r}\left(M^{p}\right)$ denote the connected component of the identity.

THEOREM 1. $\operatorname{Diff}_{0}^{\infty}\left(M^{p}\right)$ is a simple group.

The proof makes use of both the theorem of Epstein [4] that the commutator subgroup of $\operatorname{Diff}_{0}\left(M^{p}\right)$ is simple, and of the result of M. Herman [9] which gives the case $M^{p}$ is a $p$-torus.

THEOREM 2. $B \bar{\Gamma}_{p}^{\infty}$ is $(p+1)$-connected, where $B \bar{\Gamma}_{p}^{\infty}$ is the classifying space for framed, codimension $p, C^{\infty}$, Haefliger structures.

The more usual notation is $F \Gamma_{p}^{\infty}=B \bar{\Gamma}_{p}^{\infty}$. Haefliger proved [6] that $B \bar{\Gamma}_{p}^{r}$ is $p$-connected for $1 \leqq r \leqq \infty$; Mather proved that $B \bar{\Gamma}_{1}^{\infty}$ is 2-connected.

Theorem 2 means that two $C^{\infty}$ foliations of a manifold coming from nonsingular vector fields are homotopic as Haefliger structures if and only if the normal bundles are isomorphic.

Theorems 1 and 2 are proven by showing they are related; $\mathrm{cf}$. Theorem 4 for a statement of a relationship.

Corollary. $P_{1}^{[p / 2]}$ is nontrivial in $H^{*}\left(B \Gamma_{p}^{\infty} ; \boldsymbol{R}\right)$ where $P_{1}$ is the first real Pontrjagin class of the normal bundle to the canonical Haefliger structure.

AMS (MOS) subject classifications (1970). Primary 57D30, 57D50. 
Thus, Bott's vanishing theorem [1], which says real Pontrjagin classes in $B \Gamma_{p}^{r}(r \geqq 2)$ vanish above dimension $2 p$, gives a sharp bound on dimensions.

This corollary in the case $p=2$ follows easily from Theorem 2 .

For higher codimensions, product foliations then yield examples.

THeOREM 3. $B \bar{\Gamma}_{p}^{0}$ is contractible.

Again, Mather proved this when the codimension is one.

This means topological Haefliger structures are completely determined up to homotopy by their normal micro-bundles.

Theorem 3 implies that Bott's vanishing theorem is quite false in the topological case-any normal micro-bundle is the normal micro-bundle for a topological foliation. In fact, if the micro-bundle is differentiable, it even admits a Haefliger structure Lipschitz close to being differentiable.

A little background and notation is necessary before the statement of the more general relationships. Let $G$ be a topological group. Let $G_{\delta}$ be $G$ with discrete topology. Then the map $G_{\delta} \rightarrow G$ is a continuous map which has a homotopy-theoretic fiber $\bar{G}$. $\bar{G}$ is also a topological group: the explicit construction for $\bar{G}$ is the space of paths $\alpha$ in $G$ ending at the identity $e=\alpha(1)$, with discrete topology on $\alpha(0)$. Then multiplication is pointwise. There are maps, now,

$$
\bar{G} \rightarrow G_{\delta} \rightarrow G \rightarrow B \bar{G} \rightarrow B G_{\delta} \rightarrow B G,
$$

and any two consecutive arrows define a fibration.

$B G$ is the classifying space for $G$-bundles. $B G_{\delta}$ classifies flat $G$-bundles: for instance, $B \operatorname{Diff}^{\infty}\left(M^{n}\right)_{\delta}$ has an associated $M$-bundle, with discrete structure group: i.e., a $C^{\infty}$ foliation transverse to the fibers of the bundle. Thus, $B$ Diff ${ }^{\infty}\left(M^{p}\right)_{\delta}$ classifies "foliated $M^{p}$-bundles". Finally, $B \bar{G}$ classifies $G$-bundles with a flat structure, together with a global trivialization defined (up to homotopy); e.g. $B \overline{\operatorname{Diff}}{ }^{\infty}\left(M^{p}\right)$ classifies "foliated $M^{p_{-}}$ products".

Let $\operatorname{Diff}_{K}\left(\boldsymbol{R}^{p}\right)$ be the group of diffeomorphisms of $\boldsymbol{R}^{p}$ with compact support. Then again, $B \overline{\operatorname{Diff}}_{K}^{r}\left(\boldsymbol{R}^{p}\right) \times \boldsymbol{R}^{p}$ has a foliation of codimension $p$ transverse to the $\boldsymbol{R}^{p}$-factors. Thus, there is a classifying map

$$
B \overline{\operatorname{Diff}}_{K}^{r}\left(\boldsymbol{R}^{p}\right) \times \boldsymbol{R}^{p} \rightarrow B \bar{\Gamma}_{p}^{r} .
$$

(The image is in $B \bar{\Gamma}_{p}^{r}$ since there is a natural trivialization of the normal bundle to the foliation.)

The foliation agrees with the trivial, product foliation in a neighborhood of $\infty$ in the $\boldsymbol{R}^{p}$ factors. Thus, one obtains a map of the $p$-fold suspension of $B \overline{D i f f}_{K}^{r} \boldsymbol{R}^{p}$,

$$
S^{p}\left(B \overline{\operatorname{Diff}}_{K}^{r} \boldsymbol{R}^{p}\right) \rightarrow B \bar{\Gamma}_{p}^{r} .
$$


This defines an adjoint map $B \overline{\operatorname{Diff}}{ }_{K}^{r}\left(\boldsymbol{R}^{p}\right) \rightarrow \Omega^{p}\left(B \bar{\Gamma}_{p}^{r}\right)$ to the $p$-fold loop space of $B \bar{\Gamma}_{p}^{r}$.

THEOREM 4. The map $B \overline{D i f f}_{K}^{r}\left(\boldsymbol{R}^{p}\right) \rightarrow \Omega^{p}\left(B \bar{\Gamma}_{p}^{r}\right)$ induces an isomorphism on homology.

This theorem is due to Mather in the case $p=1$.

The map is certainly not a homotopy equivalence since $\pi_{1}\left(B \overline{\mathrm{Diff}}_{K}^{r} \boldsymbol{R}^{p}\right)$ is highly nonabelian while $\pi_{1}\left(\Omega^{p} B \bar{\Gamma}_{p}^{r}\right)=\pi_{p+1}\left(B \bar{\Gamma}_{p}^{r}\right)$ is abelian.

Similarly, there is a map $B \operatorname{Diff}^{r}\left(M^{p}\right) \times M^{p} \rightarrow B \Gamma_{p}^{r}$ which is a lifting of the classifying map for the tangent bundle of $M^{p}$, so there is a commutative diagram

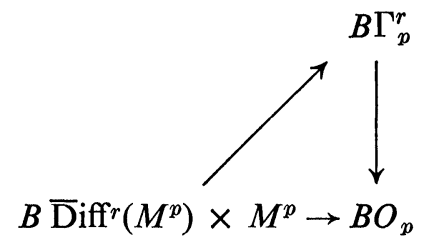

Let $X$ be the space of liftings of the classifying map for $T\left(M^{p}\right)$ in $B O_{p}$ to $B \Gamma_{p}^{r}$. Then we have a map $B \overline{\operatorname{Diff}}^{r}\left(M^{p}\right) \rightarrow X$.

THEOREM 5. The map

$$
B \overline{\operatorname{Diff}} r\left(M^{p}\right) \rightarrow X
$$

induces an isomorphism on homology.

Again, this is not a homotopy equivalence since $\pi_{1}(X)$ is abelian.

For the case $r=0$, we assume $M^{p}$ is a differentiable manifold.

Corollary. (a) $B \operatorname{Homeo}\left(M^{p}\right)$ is acyclic, where $\operatorname{Homeo}\left(M^{p}\right)=$ $\operatorname{Diff}^{0}\left(M^{p}\right)$ is the group of homeomorphisms of $M^{p}$.

(b) The map $B$ Homeo $\left(M^{p}\right)_{\delta} \rightarrow B$ Homeo $\left(M^{p}\right)$ induces an isomorphism on homology.

This corollary is implied by Theorems 3 and 5. Cf. Mather [13], who showed $B \mathrm{Homeo}_{K}\left(\boldsymbol{R}^{p}\right)_{\delta}$ is acyclic.

COROLLARY. The following groups are isomorphic, where $k$ is the first positive integer such that one of them is nontrivial:

(i) $H_{k}\left(B \operatorname{Diff}^{r}\left(M^{p}\right) ; Z\right)$,

(ii) $H_{k}\left(B \overline{\operatorname{Diff}}_{K}^{r}\left(\boldsymbol{R}^{p}\right) ; Z\right)$,

(iii) $H_{k+p}\left(B \Gamma_{p}^{r} ; Z\right)$.

CONJECTURE. This first $k$ is $p+1$, for $r=\infty$.

Mather's theorem [11] shows this for $p=1$. Bott and Haefliger showed 
that all differentiable characteristic classes (in some sense) vanish below this dimension, $H_{2 p+1}\left(B \bar{\Gamma}_{p}^{r} ; Z\right)$ [2], [3].

In [16] I sketched examples showing there is a surjective homomorphism

$$
H_{3}\left(B \bar{\Gamma}_{1}^{\infty} ; Z\right) \rightarrow R,
$$

using the Godbillon-Vey invariant gv [5]. Recently I have extended this to arbitrary codimension, so there is a surjective homomorphism

$$
H_{2 p+1}\left(B \bar{\Gamma}_{p}^{\infty} ; Z\right) \rightarrow \boldsymbol{R} \text {. }
$$

\section{BIBLIOGRAPHY}

1. R. Bott, On a topological obstruction to integrability, Proc. Int. Congress Nice, 1970, 27-36.

2. R. Bott and A. Haefliger, On characteristic classes of $\Gamma$-foliations, Bull. Amer. Math. Soc. 78 (1972), 1039-1044.

3. - Continuous cohomology and characteristic classes, (to appear).

4. D. B. A. Epstein, The simplicity of certain groups of homeomorphisms, Compositio Math. 22 (1970), 165-173. MR 42 \#2491.

5. C. Godbillon and J. Vey, Un invariant des feuilletage de codimension 1, C.R. Acad. Sci. Paris Sér. A-B 273 (1971), A92-A95. MR 44 \#1046.

6. A. Haefliger, Feuilletages sur les variétés ouvertes, Topology 9 (1970), 183-194. MR 41 \#7709.

7. - Homotopy and integrability, Manifolds-Amsterdam 1970 (Proc: Nuffic Summer School), Lecture Notes in Math., vol. 197, Springer, Berlin, 1971, pp. 133-163. MR 44 \#2251.

8. - Sur les classes characteristiques des feuilletages, Séminaire Bourbaki, No. 412, June 1972.

9. M. Herman, Simplicité du groupe des difféomorphismes de classe $C^{\infty}$, isotopes à l'identité, du tore de dimension n, C.R. Acad. Sci. Paris Sér. A-B 273 (1971), A232A234. MR 44 \#4788.

10. M. Herman and F. Sergeraert, Sur un théorème d'Arnold et Kolmogorov, C.R. Acad. Sci. Paris Sér. A-B 273 (1971), A409-A411. MR 44 \#7586.

11. J. Mather, On Haefliger's classifying space. I, Bull. Amer. Math. Soc. 77 (1971), 1111-1115. MR 44 \#1047.

12. - On Haefliger's classifying space. II: Approximation theorems, (preprint).

13. - The vanishing of homology of certain groups of homeomorphisms, Topology 10 (1971), 297-298. MR 44 \#5973.

14. - Integrability in codimension 1, Comment. Math. Helv. (to appear).

15. W. Thurston, Noncobordant foliations of $S^{3}$, Bull. Amer. Math. Soc. 78 (1972), 511-514. MR 45 \#7741.

16. —_ Variation of the Godbillon-Vey invariant in higher codimension, (to appear).

Department of Mathematics, University of California, Berkeley, California 94720

School of Mathematics, Institute for Advanced Study, Princeton, New JERSEY 08540

Current address: Department of Mathematics, Massachusetts Institute of Technology, Cambridge, Massachusetts 02139 